\title{
Genes de virulencia de Escherichia coli detectados en muestras diarreicas de niños de la Región Lambayeque - Perú
}

Yacarini-Martínez Antero Enrique* 1,a,b; Arriaga-Deza Emma Vanesa 2,3,c; Alvarado-Pineda Rosa Liliana 1,4,a,d; Fupuy-Chung Jorge Antonio ${ }^{1, e}$

\section{RESUMEN}

Objetivo: Detectar genes asociados a factores de virulencia de Escherichia coli aisladas de muestras diarreicas de niños menores de cinco años con el empleo de PCR Multiplex.

Materiales y métodos: Se realizó un trabajo descriptivo, transversal, de una sola cohorte de muestras diarreicas de niños menores de cinco años colectadas desde enero 2014 a marzo 2015. Se usaron primers específicos para genes de los seis patotipos causantes de diarreas infantiles: gen daaD (Escherichia coli difusamente adherente - DAEC), gen aggR (Escherichia coli enteroagregativa - EAEC), gen eaeA(Escherichia coli enteropatógena - EPEC), gen stx (Escherichia coli productora de toxina Shiga - STEC), gen ipaH (Escherichia coli enteroinvasiva - ElEC)y gen st (Escherichia coli enterotoxigénica - ETEC).

Resultados: Se aislaron 106 cepas de Escherichia coli en las que se encontraron genes de virulencia analizados en el 37,74 \% $(40 / 106)$ de las mismas. El grupo etario más afectado con la presencia de estos genes fue el comprendido entre 1-2 años $(48,6 \%)$.

Conclusiones: El gen daaD del patotipo DAEC presentó la mayor distribución en un 16,98 \%; así mismo la detección de los genes de virulencia específicos podría ayudar a tratar de manera adecuada y oportuna un episodio de diarrea aguda infantil.

Palabras clave: Escherichia coli; Genes; Factores de virulencia; Diarrea (Fuente: DeCS BIREME).

\section{Escherichia coli virulence genes detected in diarrheal samples of children from the Lambayeque region - Peru}

\author{
ABSTRACT
}

Objective: This study aimed to detect, by multiplex PCR, genes associated with virulence factors of Escherichia coli de isolated from diarrheal samples of children under 5 years of age.

Materials and methods: A descriptive, cross-sectional, single-cohort study, in which diarrheal samples from children under five years of age collected from January 2014 to March 2015 were analyzed. Specific primers for detecting the genes of the six pathotypes that cause childhood diarrhea were used: daaD gene (diffusely adherent Escherichia coli - DAEC), aggR gene (Enteroaggregative Escherichia coli - EAEC), eaeA gene (enteropathogenic Escherichia coli - EPEC), stx gene (Shiga toxin-producing Escherichia coli - STEC), ipaH gene (Enteroinvasive Escherichia coli - EIEC) and st gene (Enterotoxigenic Escherichia coli - ETEC).

Results: Virulence genes were found in $37.74 \%$ ( 40/106) of the 106 Escherichia coli isolated strains. The 1-to 2-year-old age group was the most affected with these genes (48.6\%).

Conclusions: The daaD gene of the DAEC pathotype showed the greatest distribution (16.98 \%). The detection of specific virulence genes could help to treat an episode of acute childhood diarrhea in an appropriate and timely manner.

Keywords: Escherichia coli; Genes; Virulence factors; Diarrhea (Source: MeSH NLM).

1. Universidad Católica Santo Toribio de Mogrovejo, Facultad de Medicina. Chiclayo, Perú.

2. Universidad de San Martín de Porres, Facultad de Medicina Humana. Chiclayo, Perú.

3. Dirección de Investigación Hospital Regional Lambayeque. Chiclayo, Perú.

4. Laboratorio Referencial de Salud Pública. Chiclayo, Perú.

a. Maestro en Ciencias con mención en Microbiología.

b. Docente adscrito al Departamento de Ciencias de la Salud.

c. Licenciada en Biología, Microbiología y Parasitología.

d. Responsable del Laboratorio de Enteropatógenos.

e. Maestro en Ciencias con mención en Ecología y Conservación, Docente adscrito al Departamento de Ciencias de la Salud.

* Autor corresponsal. 


\section{INTRODUCCIÓN}

Las enfermedades diarreicas constituyen un problema de salud pública en el mundo, que causan morbilidad y mortalidad en niños menores de 5 años ${ }^{(1,2)}$.

Entre los agentes etiológicos clásicos que producen un cuadro diarreico importante en los niños se encuentra la Escherichia coli, de la cual, hasta la fecha, se han descrito 6 tipos patogénicos: $E$. coli enterotoxigénica (ETEC), E. coli enteroinvasiva (EIEC), E. coli enteropatogénica (EPEC), E. coli enterohemorrágica (ECEH), $E$. coli enteroagregativa (ECEA) y $E$. coli adherente difusa (DAEC). Cada uno de los tipos patogénicos presentan diferencias a nivel de virulencia, interacción con la mucosa intestinal, síndrome clínico asociado, epidemiología y serotipos basados en los antígenos $\mathrm{O}$ y $\mathrm{H}^{(3-6)}$.

No hay estudios al respecto sobre la base de la problemática de salud en la Región Lambayeque por lo que queda un vacío de información acerca de la frecuencia con que los diferentes patotipos de $E$. coli producen estos cuadros. Este estudio se enmarca en las prioridades de investigación de la región que está asociada a infecciones de origen entérico; tiene por finalidad determinar la distribución etiológica de los patotipos de $E$. coli diarreogénica en niños menores de cinco años con diarrea aguda.

\section{MATERIALES Y MÉTODOS}

\section{Diseño y población}

Estudio observacional descriptivo, de corte transversal. Se incluyeron las muestras de pacientes menores de cinco años con diarrea aguda, acuosa persistente o disentérica, y se excluyeron a los que fueron tratados con algún tipo de antibiótico.

Se trabajó con 106 cepas de E. coli aisladas por coprocultivo, de muestras de diarreas de niños menores de cinco años, colectadas desde enero 2014 a marzo 2015, procedentes de 9 establecimientos de salud de la Región Lambayeque, que fueron identificadas mediante pruebas de diferenciación bioquímica (LIA, TSI, citrato y SIM).

\section{Variables y mediciones}

Para la detección de los patotipos de $E$. coli diarreogénica se utilizó la prueba de PCR Multiplex en tiempo real (mRT$\mathrm{PCR}$ ) en un pool de 5 colonias "lactosa-positivas", a partir de la placa de agar "Mac Conkey", para la búsqueda de los seis genes de virulencia relacionados con cada uno de los patotipos de $E$. coli de interés para la investigación, previamente estandarizada y validada. Se utilizaron primers diseñados específicamente para amplificar, lo que permitió la identificación de los amplicones al emplear un fluoróforo SYBR Green, el cual, al unirse a las cadenas de $A D N$ de doble hebra emite fluorescencia, que aumenta a medida que el producto se acumula con cada ciclo de la amplificación.

\section{Análisis estadístico}

Se emplearon tablas de frecuencias, para resumir la información del porcentaje de frecuencia de cada gen así como de cada patotipo. Los datos se clasificaron por centro de salud de origen de la muestra, y se categorizaron por grupos de edad (menor a un año, de 1 a 2, de 2 a 3 y de 3 a 5 años). Por último, se han utilizado gráficos de barras (\%) para que la información sea más fácil de interpretar.

\section{Consideraciones éticas}

Se guardaron la reserva y confidencialidad de toda la información a la que se tuvo acceso y se respetaron los principios éticos de la investigación científica, así como los principios universales de bioseguridad.

\section{RESULTADOS}

Se analizaron 106 cepas que cumplieron con los criterios de inclusión, provenientes de 9 establecimientos de Salud, los hospitales Belén y Las Mercedes, y los Centros de Salud José Leonardo Ortiz, José Olaya, José Quiñones, La Pradera, San Antonio, La Victoria y Cruz de la Esperanza.

Para la determinación de las $E$. coli diarreogénicos (DEC) se usó el mRT-PCR, el cual fue implementado en el Laboratorio de Infecciones Pediátricas de la Universidad Peruana Cayetano Heredia en Lima. Este PCR utiliza fluorescencia para determinar la temperatura de denaturación de cada amplicón basado en la disociación del SYBR Green ${ }^{(3,4)}$.

Los primers o cebadores han sido diseñados para reconocer simultáneamente nueve genes en una sola reacción: aggR(EAEC), st1, st2, lt(ETEC), eae(EPEC), eae, stx1, stx2 (STEC), ipaH(EIEC), y daaD (DAEC).

Luego del análisis por PCR Multiplex se encontró que el $16,98 \%$ de las cepas presentaron el gen daaD perteneciente al patotipo DAEC; el 7,55\%, el gen aggR que corresponde al patotipo ECEA; el $1,89 \%$ de las muestras tiene el gen eaeA que pertenece al patotipo EPEC; 11,32 \%de las cepas presenta el gen st del patotipo ETEC; y en $62,26 \%$ de las cepas no se detectó gen asociado a ningún patotipo de $E$. coli evaluado (Tabla 1).

El gen daaD se encontró con mayor prevalencia en los 9 establecimientos ( $45 \%$ ) en relación a los genes detectados (Figura 1).

Lo anteriormente detallado nos indica que solo un $37,74 \%$ (40) de las 106 cepas aisladas respondieron a los patotipos de Escherichia coli analizados. 
Tabla 1. Genes de factores de virulencia encontrados para cada patotipo de $E$. coli

\begin{tabular}{|llrr|}
\hline Patotipo & Gen & $\mathbf{n}$ & \% \\
\hline DAEC & daaD & 18 & 16,98 \\
EAEC & aggR & 8 & 7,55 \\
EPEC & $e a e A$ & 2 & 1,89 \\
ETEC & St & 12 & 11,32 \\
STEC & Stx & 0 & 0,00 \\
EIEC & $i p a H$ & 0 & 0,00 \\
No detectado & & 66 & 62,26 \\
\hline Total general & & 106 & 100.00 \\
\hline
\end{tabular}

En esta tabla se aprecian los porcentajes obtenidos para cada gen asociado a los factores de virulencia de cada patotipo de Escherichia coli aislada.

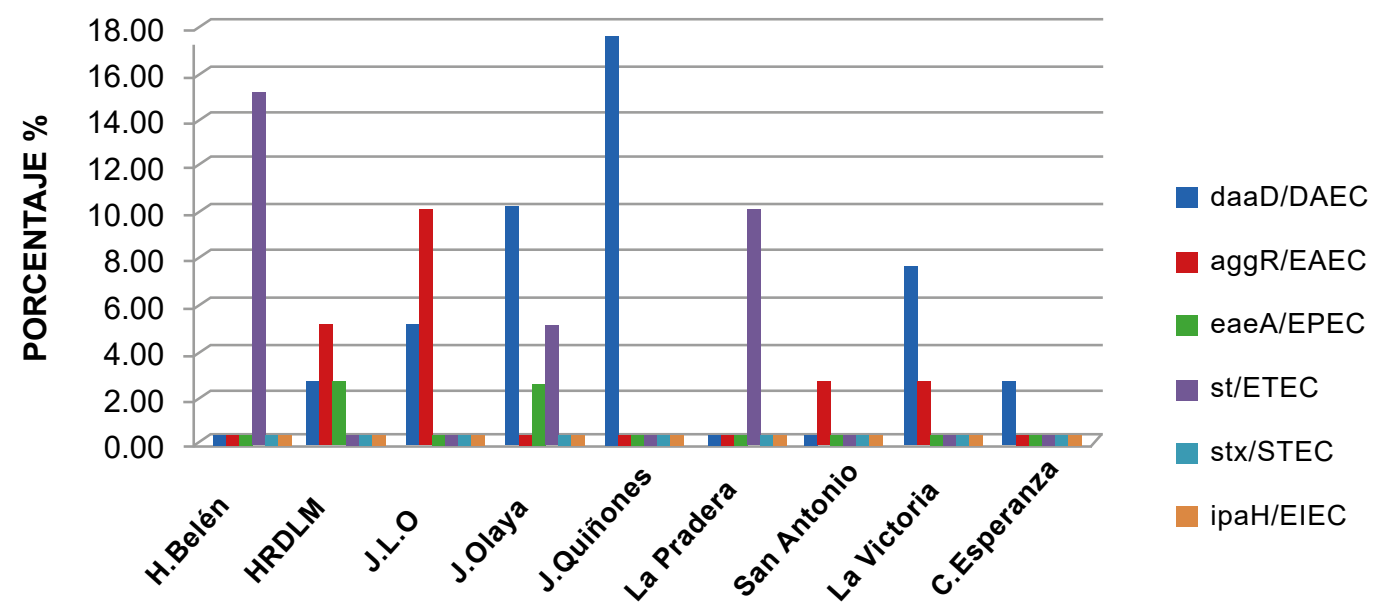

\section{LUGAR DE PROCEDENCIA}

Figura 1. Distribución de patotipos de $E$. coli según su procedencia

En esta figura se aprecia la distribución de patotipos de Escherichia coli de acuerdo a la procedencia de las muestras analizadas, en los que se especifica el gen de virulencia asociado a cada uno de dichos patotipos.
Según grupo etario los 4 genes analizados se encontraron en un 48,6\% entre las edades de 1 a 2 años, seguido de un $28,6 \%$ entre las edades de 3 a 5 años. Mientras que los grupos de menores de un año y el grupo de 2 a 3 años presentaron valores de $15 \%$ y $7,8 \%$, respectivamente (Tabla 2 y figura 2 ). 
Genes de virulencia de Escherichia coli detectados en muestras diarreicas de niños de la Región Lambayeque - Perú

Tabla 2. Distribución de genes asociados a factores de virulencia de patotipos de $E$. coli según grupo etario

$\begin{array}{lccccccc}\text { Grupos de edad } & \begin{array}{c}\text { daaD/DAE } \\ \text { C }\end{array} & \begin{array}{c}\text { aggR/EAE } \\ \text { C }\end{array} & \begin{array}{c}\text { eaeA } \\ \text { /EPEC }\end{array} & \begin{array}{c}\text { st } \\ \text { /ETEC }\end{array} & \begin{array}{c}\text { Stx } \\ \text { /STEC }\end{array} & \begin{array}{c}\text { ipaH } \\ \text { /EIEC }\end{array} & \text { TOTAL } \\ \text { Menor a 1 año } & 2,9 & 6,1 & 0,0 & 6,0 & 0,0 & 0,0 & 15,0 \\ \text { 1 a 2 años } & 22,9 & 8,7 & 3,4 & 13,5 & 0,0 & 0,0 & 48,6 \\ \text { 2 a 3 años } & 5,3 & 0,00 & 2,5 & 0,00 & 0,0 & 0,0 & 7,8 \\ \text { 3 a 5 años } & 25,7 & 0,3 & 0,00 & 2,6 & 0,0 & 0,0 & 28,6 \\ \text { TOTAL } & 56,8 & 15,2 & 5,9 & 22,1 & 0,0 & 0,0 & 100,0\end{array}$

En esta tabla se puede evidenciar los grupos etarios pediátricos afectados por cada patotipo de Escherichia coli y su respectivo gen asociado al factor de virulencia.

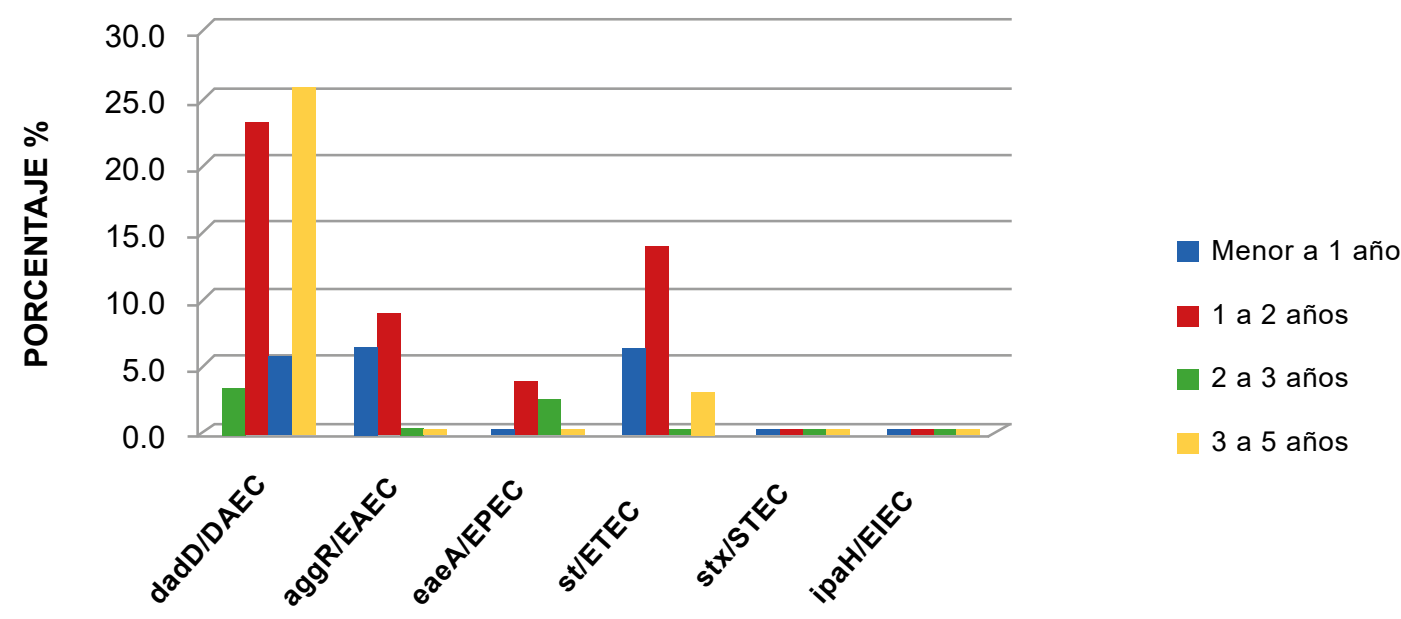

GEN / PATOTIPO Escherichia coli

Figura 2. Distribución de genes asociado a factores de virulencia de patotipos de $E$. coli según grupo etario

\section{DISCUSIÓN}

La diarrea continúa siendo la tercera causa de muerte en niños menores de 5 años, pese a los avances recientes en el manejo y prevención de esta enfermedad. Es causada por múltiples patógenos, sin embargo, la prevalencia de cada uno varía según el grupo etario, la zona geográfica y el escenario donde se registran los casos, ya sea comunitario u hospitalario ${ }^{(7-9)}$.

Los patógenos más relevantes en salud pública son los asociados con mayor carga de enfermedad, severidad, complicaciones y mortalidad. En nuestro medio, el
Norovirus, Campylobacter y los diferentes patotipos de $E$. coli diarreogénicas (DEC) son los patógenos más prevalentes a nivel comunitario en niños $(7,10,11)$.

A pesar de que el coprocultivo es el método de diagnóstico de mayor uso para la detección de $E$. coli, el crecimiento de este microorganismo no es adecuadamente interpretado, porque se considera como una bacteria de flora normal y se omite el verdadero potencial patogénico de la misma $(5,10,12)$.

Por lo anteriormente mencionado, los diferentes patotipos de $E$. coli, causantes de cuadros diarreicos, no siempre 
pueden ser detectados mediante diagnóstico microbiológico convencional, ni por diagnóstico serológico, debido a que este tipo de pruebas no demuestran de manera específica la presencia de esta bacteria ${ }^{(2,11)}$.

A pesar de que en los laboratorios clínicos, por ejemplo, la identificación de EPEC se basa en la determinación de serotipos específicos por técnicas de aglutinación que utilizan antisueros $\mathrm{O}$ y $\mathrm{H}$; actualmente, la identificación del gen de intimina (eaeA) por PCR es el método diagnóstico de elección para EPEC ${ }^{(10,13)}$.

Luego de procesar, aislar e identificar $E$. coli en las muestras estudiadas, se realizó el estudio molecular con la prueba de la reacción en cadena de la polimerasa (PCR) variante Multiplex, con la que se determinó que el 16,98\% de las cepas portan el gen daaD (patotipo DAEC), mientras que el $11,32 \%$ (gen st) corresponde al patotipo ETEC. El patotipo EAEC, identificado con el gen aggR, se encuentra en el 7,55 \% de las cepas; finalmente, solo el $1,89 \%$ de las cepas presentaron el gen eaeA correspondiente al patotipo EPEC. Ninguna de las cepas portaba genes stx e ipaH, que pertenecen a los patotipos STEC y EIEC, respectivamente. El gen daaD tuvo la mayor prevalencia ( $45 \%$ ) en comparación con los otros genes detectados dentro de los centros de salud incluidos en el estudio.

En un estudio realizado por Ochoa et al.(4), se encontraron los patotipos EAEC, EPEC, ETEC y DAEC, en donde la prevalencia promedio global de los principales patógenos en muestras de diarrea fue $\operatorname{EAEC}(9,9 \%)$, $\operatorname{EPEC}(8,5 \%)$, ETEC $(6,9 \%)$ y DAEC $(4,8 \%)$ sin embargo, este estudio señala algunas particularidades, pues en niños con diarrea y deshidratación el patógeno más frecuente fue ETEC, responsable del $20,8 \%$ de todos los episodios, seguido de $\operatorname{DAEC}(15,0 \%)$.

Este estudio nos sirve de base para el análisis respectivo de los resultados obtenidos en la presente investigación, en estudios comunitarios y con episodios de diarreas más leves, los patógenos más frecuentes fueron EPEC, EAEC y ETEC.

Por otro lado, una investigación realizada por Riveros et al. ${ }^{(6)}$, que compara los patrones de adherencia entre cepas DAEC aisladas de niños con y sin diarrea, precisa que este patotipo DAEC es el sexto grupo de $E$. coli diarreogénica reconocido y que está asociado con el gen daaD, lo que plantea una relación de asociación de episodios diarreicos controversiales, pero de mucha importancia en el desarrollo de cuadros diarreogénicos con ciertas características clínicas, lo cual también se evidencia en nuestro estudio.

A pesar de que en las investigaciones anteriores ${ }^{(4,6)}$, realizadas por separado se han encontrado datos $y$ resultados sumamente importantes en la detección de genes de patotipos diarreogénicos, la importancia y relevancia de nuestra investigación radica, en que a pesar de que no existen antecedentes de este tipo de estudios moleculares en nuestra región norte para el caso de muestras diarreogénicas, hallamos una presencia importante en un mayor porcentaje del gen daaD como factor de virulencia del patotipo DAEC sobre todo en niños de 3 a 5 años.

El análisis, en su conjunto, de todos los resultados obtenidos nos demuestra que, si bien hubo una mayor presencia del gen daaD perteneciente al patotipo DAEC, esto no descarta la importancia de realizar más estudios con un enfoque molecular y establecer una relación más amplia de cuáles los patotipos más frecuente, en función a la población pediátrica más vulnerable.

En conclusión, se determinaron genes asociados a factores de virulencia mediante el método molecular de PCR Multiplex para los patotipos de Escherichia coli asociados clínicamente a cuadros diarreicos. Así mismo, se encontró que el $16,98 \%$ de las cepas aisladas presentaron el gen daaD perteneciente al patotipo DAEC. Por otro lado, se determinó que el $11,32 \%$ de las cepas presentaron el gen st perteneciente al patotipo ETEC, el 7,55 \% de las cepas presentaron el gen $a g g R$ perteneciente al patotipo EAEC, el $1,89 \%$ de las cepas presentaron el gen eaeA perteneciente al patotipo EPEC. Se concluye además que un $62,26 \%$ de las cepas no se detectó gen asociado a ningún patotipo de Escherichia coli estudiado. El patotipo DAEC presentó mayor frecuencia en seis centros de salud y su presencia está relacionada con cuadros de diarrea acuosa sin sangre y sin leucocitos.

Al considerar que muchas veces los reportes de Escherichia coli en los coprocultivos de diferentes tipos de diarrea no son tomados en cuenta; la detección de los genes de virulencia específicos de Escherichia coli podría denotar la importancia de considerar epidemiológicamente a esta bacteria y al mismo tiempo tratar de manera adecuada y oportuna un episodio de diarrea aguda infantil sin causa aparente.

Agradecimientos: Dra. Theresa Ochoa Woodell. Jefa de Laboratorio de Infectología Pediátrica. UPCH. Lima Blgo. David Durand Vara. Investigador del Laboratorio de Infectología Pediátrica. UPCH. Lima

\section{REFERENCIAS BIBLIOGRÁFICAS}

1. Alfonso González MJ, González Sosa N, López Banasco N. Viabilidad y características culturales, tintoriales, morfológicas y bioquímicas de una colección bacteriana. Rev Cub Med. 2015;44(1):105-11

2. Alvarado R. Enfermedad diarreica asociada a Escherichia 
coli Enteropatógena Clásica (EPEC), Enteroinvasiva (EIEC) en pacientes pediátricos en dos Hospitales de Lima [Tesis para optar el grado de maestría en microbiología]. Lima: Universidad Nacional Mayor de San Marcos; 2008.

3. Barletta F, Ochoa T, Mercado E, Ruiz J, Ecker L, Lopez G, et al. Quantitative real-time polymerase chain reaction for enteropathogenic Escherichia coli: A tool for investigation of asymptomatic versus symptomatic infections. Clinical Infectious diseases advance. Clin Infect Dis. 2011; 53(12): 1223-1229.

4. Ochoa T, Mercado E, Durand D, Rivera F, Mosquito S, Contreras C, et al. Frecuencias y patotipos de Escherichiacoli Diarrogénica en niños peruanos con y sin diarrea. Rev Peru Med Exp Salud Pública. 2011; 28(1): 13-20.

5. Riveros M, Ochoa TJ. Enteropatógenos de importancia en salud pública. Rev Peru Med Exp Salud Pública. 2015; 32(1):157-64.

6. Riveros M, Barletta F, Cabello M, Durand D, Mercado E, Contreras C, et al. Patrones de adherencia de cepas de Escherichia coli difusamente adherente (DAEC) provenientes de niños con y sin diarrea. Rev Peru Med Exp Salud Pública. 2011; 28(1): 21-28.

7. Silva-Díaz H, Bustamante-Canelo O, Aguilar-Gamboa F, Mera-Villasis K, Ipanaque-Chozo J, Seclén-Bernabé E, et al. Enteropatógenos predominantes en diarreas agudas y variables asociadas en niños atendidos en el Hospital Regional Lambayeque, Perú. Horiz Med. 2017; 17(1): 38-44.

8. Toma C, Lu Y, Higa N, Nakasone N, Chinen I, Baschkier A, et al. Multiplex PCR Assay for Identification of Human Diarrheagenic Escherichia coli. J Clin Microbiol. 2003; 41(6): 2669-71.

9. Brito E, Oliveira C, Becerra E, Vasconcelos S, Da Cruz D, De Panta $F$, et al. La detección de bacterias enteropatógenos y enteroparasitarias en los pacientes con diarrea aguda en Juruti, Pará y Brasil. Rev. Pun Amoz Soude.2009;1:143-48.

10. Contreras C. Asociación de variabilidad genética y fenotípico de Escherichia coli enteropatógena (EPEC) con cuadros de diarrea en niños menores de un año [Tesis para optar el grado de Maestría en Biología Molecular]. Lima: Universidad Nacional Mayor de San Marcos; 2010.

11. Manrique F, Billón D, Bello S, Ospina J. Agentes causantes de

diarreas en niños menores de 5 años en Tunja, Colombia. Rev Salud Pública. 2006; 8(1):88-97.

12. Vidal JE, Canizález-Román A, Gutiérrez-Jiménez J, NavarroGarcía F. Patogénesis molecular, epidemiología y diagnóstico de Escherichia coli enteropatógena. Salud Públ Méx. 2007; 49(5): 276-86

13. Contreras CA, Ochoa TJ, Ruiz J, Lacher DW, Durand D, DebRoy C. Genetic diversity of locus of enterocyte effacement genes of enteropathogenic Escherichia coli isolated from Peruvian children. J Med Microbiol. 2012;61(8):1114-20.

Fuentes de financiamiento:

Este artículo ha sido financiado por los autores.

Conflictos de interés:

Los autores declaran no tener ningún conflicto de interés.

Correspondencia:

Antero Enrique Yacarini Martínez

Dirección: Calle Los Tulipanes Mz. B- Lote 3. Conjunto Habitacional Hans Bruning. Lambayeque, Perú.

Teléfono: 962558706

Correo electrónico: ayacarini@usat.edu.pe

Recibido: 12 de junio de 2018.

Evaluado: 19 de junio de 2018.

Aprobado: 05 de setiembre 2018.

(c) La revista. Publicado por Universidad de San Martín de Porres, Perú. (cc) ${ }_{\text {BY }}$ Licencia de Creative Commons Artículo en acceso abierto bajo términos de Licencia Creative Commons Atribución 4.0 Internacional. (http://creativecommons.org/licenses/by/4.0/)

ORCID iDs

Yacarini Martínez Antero Enrique Alvarado Pineda Rosa Liliana Arriaga Deza Emma Vanesa Fupuy Chung Jorge Antonio https: / /orcid.org/0000-0003-4716-4371 https://orcid.org/0000-0002-2072-3287

https://orcid.org/0000-0002-5384-6152

https://orcid.org/0000-0003-2007-1490 